\title{
Detection Threshold and Mechanical Impedance of the Hand in a Pen-Hold Posture
}

\author{
Ali Israr ${ }^{1}$, Seungmoon Choi ${ }^{1,2}$ and Hong Z. Tan ${ }^{1}$ \\ ${ }^{1}$ Haptic Interface Research Laboratory \\ Purdue University \\ West Lafayette, IN, USA \\ ${ }^{2}$ Currently at Virtual Reality and Perceptive Media Laboratory \\ POSTECH \\ Pohang, Kyungbuk, Republic of Korea \\ israr@purdue.edu, choism@postech.ac.kr, hongtan@purdue.edu
}

\begin{abstract}
We report position and force detection thresholds for sinusoidal waveforms in the frequency range 10-500 $\mathrm{Hz}$ delivered through a stylus. The participants were required to hold the stylus in a way similar to that of holding the stylus of a force-feedback device. A minishaker moved the stylus along its length so that the majority of vibrations were presented tangentially to the skin of the hand. The measured position thresholds decreased initially with an increasing stimulus frequency and formed a $U$-shaped curve in the high frequency region. The thresholds of high frequency vibrations were lower than those reported previously for vibrations that were perpendicular to the skin, but were similar to the thresholds reported earlier using vibrations that were tangential to the skin. A similar force threshold curve was obtained using a force sensor attached to one end of the stylus. Mechanical impedance of the skin derived from velocity estimates and force measurements indicated that the skin and tissues in the hand holding the stylus can be modeled with mass-, damper- and spring-like elements. A comparison of the mechanical impedance from the present study with those reported previously showed similar results for vibrations delivered in the tangential and normal directions to the skin.
\end{abstract}

Index Terms - detection threshold, vibrotactile stimuli, position threshold, force threshold, mechanical impedance.

\section{INTRODUCTION}

We investigated the detection thresholds for sinusoidal motions of a stylus held by the hand. Several of our previous studies had required the availability of detection thresholds for the human hand while holding the stylus of a force-feedback haptic device, so that the perceived intensity of proximal stimuli could be estimated [1-5]. To the best of our knowledge, such data are not yet available in the literature. The majority of previous studies on displacement detection thresholds have applied well-controlled stimuli to the skin surface of a passively-supported hand (e.g., index fingerpad or thenar eminence) in a direction that is perpendicular to the skin (see, for example, [6]; and a review [7]). A recent study by Brisben et al. [8] found lower detection thresholds for vibrations transmitted through a hand-held tool in a direction tangential to the skin of the palm. It is well known that many factors, such as contact area, contact force, the use of a rigid surround, and skin temperature can affect the detection threshold levels. It follows that in order to assess the perceived intensity of signals experienced by a hand holding the stylus of a force-feedback device, it is necessary to estimate detection thresholds using the same hand posture under similar contact conditions.

A question relevant to this study was whether detection thresholds should be specified in force and/or displacement for force-feedback devices that are widely used in haptics research. Whereas most sensory substitution devices such as tactile aids for the hearing impaired are position displays [9, 10] (and therefore it makes sense to specify thresholds in displacements), almost all haptic interfaces for teleoperation and virtual environments are force displays [11, 12] (and we are not aware of any data on force threshold except for a previous study of our own [13]). In the present study, we measured both acceleration and force data at human detection threshold levels and studied their relationship.

The second objective of the present study was to estimate the mechanical impedance of the human hand while holding the stylus of a force-feedback device in a pen-hold posture. Mechanical impedance is an important measure relating force to position (and/or its derivatives). Numerous previous studies have investigated the mechanical impedance properties of the human skin for developing biomechanics models that can guide the design of new haptic devices [14-18]. In the present study, we first determined the mechanical impedance of our apparatus in unloaded condition, and then in loaded condition with the human hand as the load. The difference between the mechanical impedance estimates from the loaded and unloaded conditions provided an estimate of the impedance due to skin contact alone (cf. [18]).

In the rest of this section, we review the literature on detection threshold and mechanical impedance in Sec. 1.A and 1.B, respectively. We then present the methods used in the psychophysical experiments in Sec. II. Experimental results are shown in Sec. III. We discuss the results in Sec. IV, followed by conclusions in Sec. V.

\section{A. Detection Threshold}

Detection threshold is defined as the minimum stimulus intensity that is barely perceivable by a human observer. It is one of the most basic measures of human perception. Previous psychophysical studies have shown that the human detection threshold curve for sinusoidal vibrotactile displacement variations is composed of two main segments when plotted against stimulation frequency [7]. The low-frequency 
segment, associated with the non-Pacinian channels, is essentially flat. The high frequency segment, associated with the Pacinian channels, decreases rapidly and then increases again at the most sensitive frequency, forming a U-shaped curve. The shape of the low- and high-frequency curves and the most sensitive frequency vary depending on experimental conditions such as contact area, contact force, contact location, temperature of the skin, use of a rigid surround, stimulus duration, the participant's age, etc. In general, the most sensitive frequency is in the range $200-300 \mathrm{~Hz}$.

Bolanowski et al. measured detection thresholds in terms of displacement on the glabrous skin of thenar eminence with vibrations generated by a minishaker that were normal to the skin [6]. The vibrations were confined in the area of stimulation by an annulus surround and the temperature of the skin was kept at $30^{\circ} \mathrm{C}$. The results of their study, representing a typical threshold curve, are as follows. The threshold curve was flat from 0.4 to $3 \mathrm{~Hz}$. It then decreased with a slope of -5 $\mathrm{dB}$ per octave from 3 to $40 \mathrm{~Hz}$, and subsequently with a slope of $-12 \mathrm{~dB}$ per octave from 40 to $300 \mathrm{~Hz}$. The most sensitive frequency was at around $300 \mathrm{~Hz}$. The threshold then increased with a slope of about $2.7 \mathrm{~dB}$ per octave from 300 to $500 \mathrm{~Hz}$. Using a three-finger kinesthetic-to-cutaneous tactual stimulator, Israr et al. obtained similar threshold levels with vibrations presented normal to the skin of a fingerpad and without a surround around the contact [19]. Compared to Bolanowski et al.'s results, the threshold levels obtained by Israr et al. were about $9 \mathrm{~dB}$ higher for frequencies below about $20 \mathrm{~Hz}$ but were similar above $30 \mathrm{~Hz}$. The most sensitive frequency was around $200 \mathrm{~Hz}$. The increase in threshold level in the low and mid frequency region was mainly due to the lack of surround restricting vibrations on the skin [20]. For vibrations presented tangentially to the skin of a palm (in a power grip), Brisben et al. [8] showed that the detection thresholds at high frequency were lower than those reported in $[6,19]$. The slope of the threshold curve in the mid frequencies was steeper $(-15.7 \mathrm{~dB}$ per octave) than those reported in $[6,19]$, and the most sensitive frequency was between 150 and $200 \mathrm{~Hz}$. The lower threshold in [8] might have been due to a relatively large contact area in the palm and/or direction of the vibrations (tangential as supposed to perpendicular to the skin). For detection threshold expressed in terms of force or torque, a recent study of ours measured torque detection threshold when the participants held a mechanical rotary switch held between the thumb and the index finger [13]. The torque threshold curve exhibited a similar trend as the displacement threshold curves reported in [6] and [19].

In the present study, we measured displacement and force detection thresholds while the participants held a stylus connected to a minishaker in a pen-hold posture. Our results are compared to those reported in [6] and [8].

\section{B. Mechanical Impedance}

Mechanical impedance $(\bar{Z})$ is a measure of the resistance in a mechanical structure against applied vibrations $[14,17]$.
Mathematically, it is the ratio of the applied dynamic force $(\bar{F})$ to the velocity $(\bar{v})$ of structural vibration; i.e., $\bar{Z}=\bar{F} / \bar{v}$. If both the force and velocity are periodic waveforms, then the value of the mechanical impedance depends on the ratio of the amplitude of the force, $F$, and that of the velocity, $v$, of the waveforms, as well as the phase difference, $\phi$, between the waveforms; i.e. $\bar{Z}=(F / v) e^{j \phi}$.

The mechanical impedance of a structure can be decomposed into many mechanical elements including mass (inertia), damper (viscosity) and spring (stiffness). The frequency response of each element is distinct. The mass element shows a $20 \mathrm{~dB} /$ decade $(6 \mathrm{~dB} /$ octave) slope in a $\mathrm{dB}$ $\log$ plot of impedance vs. frequency, the damper a constant line, and the spring a $-20 \mathrm{~dB} /$ decade $(-6 \mathrm{~dB} /$ octave) slope. Previous studies have shown that all three mechanical elements contribute to the mechanical impedance of the human finger and other glabrous skin surfaces [14, 16-18]. In one study [17], mechanical impedance measured at several glabrous hand locations with normal vibrations showed prominent spring-like properties of the skin in response to mid-frequency vibrations and mass-like characteristics in response to high-frequency vibrations. The viscous or absorption properties were prominent in the resonant frequency region (about $80-200 \mathrm{~Hz}$ ) and in the low-frequency region. Other studies have shown that mechanical impedance varied with the grasping posture of the hand as well as the location and area of the skin contacting the stimulator (see, for example, [16]).

In the present study, a pen-shaped stylus was held by participants in a manner similar to that of holding a pen or the stylus of a force-feedback device. The stylus was excited along its length, resulting in mostly tangential vibrations on the skin surfaces of the hand holding the stylus. This was very different from most previous studies where vibrations were presented normal to the skin (although see [8, 15]). Another difference between the present and previous studies is that we applied threshold-level stimulation whereas many previous studies used suprathreshold vibrations for determination of the impedance model of the skin $[14,17]$. In the present study, the amplitudes of vibrations were very small, resulting in minute skin stretches with little energy stored and/or absorbed by the skin. It was expected that the viscous element would dominate the mechanical impedance model of the finger skin in the low $/ \mathrm{mid}$ frequencies.

\section{MEthodS}

\section{A. Apparatus}

The main component of the apparatus used in the present study was a commercially-available mini-shaker (Bruel \& Kjaer, type 4810). A spare stylus from the PHANToM 1.0A device (SensAble Technologies, Woburn, MA) was attached to the minishaker in order to simulate the use of a typical force-feedback haptic interface. An accelerometer (model 8794A, Kistler Instrument Corp., Amherst, New York) and a force sensor (model Nano 17, ATI Industrial Automation, 
Apex, North Carolina) were fastened between the minishaker and the stylus (see Fig. 1) through adapter plates. The bottom adapter below the force sensor was fastened to the threaded hole connected to the shaker actuator. The top adapter holding the accelerometer was connected to the stylus by means of a set screw. The apparatus was placed on several rubber pads inside a metal enclosure that sat above a table. The stylus protruded through a hole on top of the enclosure so that it could be grasped by a hand. An arm rest was built to support the participant's elbow and upper arm (see Fig. 2).

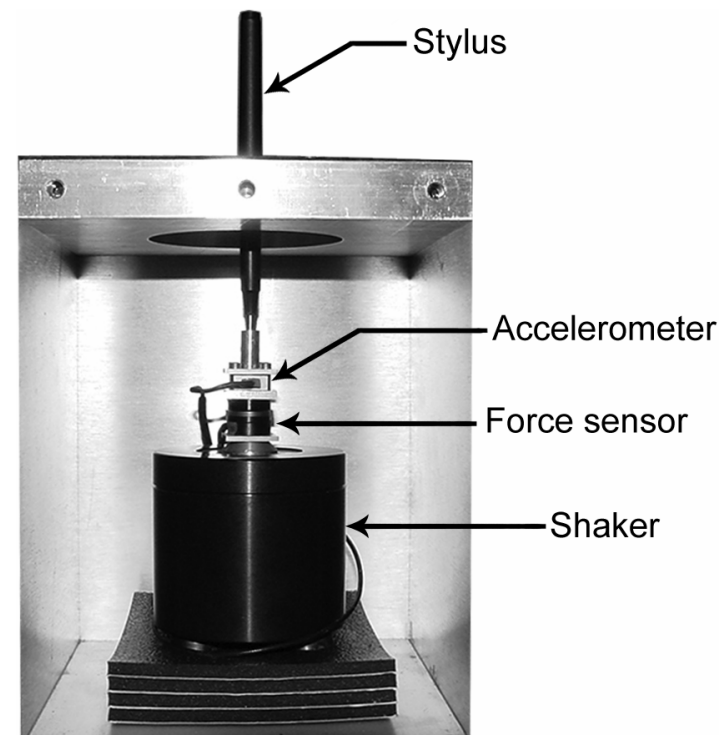

Figure 1. Shaker assembly with one of the side panels removed.

The apparatus was controlled by a data acquisition board (Nation Instruments PCI-6229, Austin, Texas) with an openloop control scheme. Command signals were generated from the board that first passed through a 16-bit Digital-to-Analog converter and then through a high-bandwidth linear audio power amplifier (model LVC 608, AE Techron Inc., Elkhart, Indiana) whose output was used to excite the minishaker vertically (along the length of the stylus). The acceleration and force data were captured by two 16-bit Analog-to-Digital converters at a sampling rate of $10 \mathrm{kHz}$. The acceleration data were integrated once and twice to obtain velocity and position estimates, respectively, for further analysis of detection threshold and mechanical impedance.

A calibration routine was performed to develop an inputoutput relationship for the apparatus at test frequencies by fitting a least-square straight-line through the controlled vibration input (in volts) and measured position data (in $\mathrm{mm}$ ) at several amplitude levels. The routine was performed without loading the apparatus and with the human hand load. The input-output relation was well represented by a straightline $\left(r^{2}>0.99\right)$ in the operating range with a significant $(p<0.0001)$ slope at each test frequency. The estimated slope and intercept of the straight-line fit were used to compensate the command signal in order to reach the desired output vibration levels. Force and position measurements were transformed into the frequency domain by taking the Fast Fourier Transform (FFT) of the measured data. Mechanical impedance was estimated by the ratio of the force/velocity amplitudes, $F / v$, and was plotted against frequency under unloaded condition. The ratio was highly linear $\left(\mathrm{r}^{2}>0.99\right)$ and a slope of $20 \mathrm{~dB} /$ decade indicated that only the inertial element was dominant between the measured force and position data (i.e., $F=m \ddot{x}$ ) under unloaded condition.

\section{B. Participants}

Five males and five females (age 22-40 years old, average 28 years old) participated in the study. Nine out of the ten participants were right-handed by self-report. Five of the participants had either participated in other haptic perception experiments in our laboratory before and/or were involved in developing the hardware/software system used in the present study. They were regarded as "experienced" users of forcefeedback and vibrotactile haptic devices. The rest of the participants were regarded as "inexperienced."

\section{Procedure}

The participant sat comfortably in front of a computer monitor with the dominant hand holding the stylus. The participant's elbow and forearm rested on the arm rest that supported a neutral wrist position (see Fig. 2). The participant was instructed to hold the stylus like holding a pen or the stylus of a PHANToM force-feedback device. Some participants practiced by using the PHANToM device (not shown) for a few minutes prior to the experiment. When the stylus was held as instructed, the vibrations transmitted through the stylus were mostly tangential to the skin in contact. The areas of the skin touching the stylus are illustrated by shaded ovals in the inset of Fig. 2 .

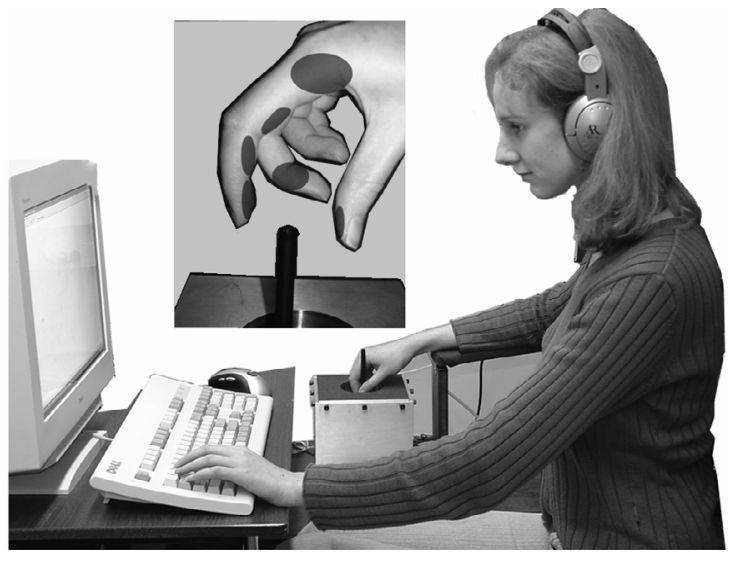

Figure 2. Experimental setup.

Seven test frequencies $(10,20,40,80,160,320$, and 500 $\mathrm{Hz}$ ) were used. They were chosen to be equally spaced on a logarithmic scale except for the highest frequency. The order of the test frequencies was randomized for each participant. The duration of the stimulus was fixed at $1 \mathrm{sec}$ with Hanning windowing (100-msec rise and fall) to reduce transient effects. 
Thresholds were obtained by a three-interval, forced-choice, one-up three-down adaptive method (See [21][22] for reviews on adaptive methods). In this method, three consecutive correct responses led to a reduction in the stimulus intensity and one incorrect response an increase, both by a predefined step size. Thresholds obtained this way correspond to the 79.4 percentile point on the psychometric function. On each trial, the participant was presented with three 1-sec long stimulus intervals with a 250 -msec inter-stimulus interval. One randomly-selected interval contained the test stimulus and the other two contained no signal. The participant's task was to indicate which one of the three intervals contained the stimulus by pressing a corresponding key ("1", "2" or "3") on the keyboard. The initial stimulus amplitude was chosen to be well above the expected detection threshold level. The step size was initially set to $4-\mathrm{dB}$ (for faster convergence) and then reduced to $1-\mathrm{dB}$ (for finer resolution) after the first three reversals (a reversal occurred if the stimulus amplitude changed from increasing to decreasing, or vice versa). A test series was terminated after 12 reversals at the $1-\mathrm{dB}$ step size. Visual and audio cues marked the start and end of each interval. The participant was required to enter a response after the end of the third interval. A new trial started right after the participant's response. Participants wore earplugs and headphones that played pink noise to block auditory cues from the apparatus.

The participants were allowed to feel the vibrations before each test condition. At the end of the experiment session, the stimulus intensity as a function of trial number was plotted. The participant was asked to repeat the series if the data failed to converge to a threshold level upon visual inspection by the experimenter. Each series took about 4-6 minutes. Participants were asked to take a 5-minute break between test conditions. The entire experiment took about 50 minutes. No correct-answer feedback was provided during the experiment.

Before the experiment, the total contact area between the participant's hand and the stylus was estimated. The participant's hand was painted with washable paint. A sheet of paper was wrapped around the stylus. The participant was then asked to hold the stylus as instructed for a few seconds, so that a good impression of the contact area was made. The contact area was estimated by scanning the sheet of paper into an AUTOCAD ${ }^{\circledR}$ program. The measured total contact area ranged from 400 to $711 \mathrm{~mm}^{2}$ (average $550 \mathrm{~mm}^{2}$ ) for all participants.

\section{Analysis of position threshold}

For each series, the last 12 reversals (six peaks and six valleys) at the $1-\mathrm{dB}$ step size were used to calculate the position detection threshold (mean of the averages of the six peak-valley pairs) and its standard deviation (from the six averages) for each participant at each test frequency.

\section{E. Analysis of force data}

Force data were processed in a manner similar to the position threshold data. The force values corresponding to the position peaks and valleys were taken as force peaks and valleys. Recall that the force measurements were captured by the force sensor that was fastened between the accelerometer and the minishaker (see Fig. 1). In order to get accurate force measurements applied on the hand holding the stylus, the sensor should have been clamped or grounded, so that the forces due to the inertia of the supporting structures could be eliminated. In most previous studies, the force sensor was placed directly in contact with the skin to measure forces due to skin contact $[14,17]$. This was not possible in the present study due to multiple contact locations between the stylus and the skin of the hand holding the stylus. Therefore, we took the approach of subtracting forces measured in unloaded condition from those in loaded condition, in order to isolate the net forces applied to the hand holding the stylus.

\section{RESULTS}

Figure 3 shows the position detection threshold curve at test frequencies used in the experiment. Each point represents the average over the ten participants and the corresponding standard error. For comparison, the figure also shows a dashed line representing the detection thresholds reproduced from Bolanowski et al. [6], and a solid line representing those from Brisben et al. [8]. The shape of the threshold curve found in the present study was very similar to those reported earlier: the threshold level decreased as frequency increased, reached a minimum at around $160-320 \mathrm{~Hz}$, and increased again at higher frequencies. The slope of the threshold curve in the frequency range $10-160 \mathrm{~Hz}$ varied from $-10 \mathrm{~dB}$ per octave $(10-20 \mathrm{~Hz})$ to $-18 \mathrm{~dB}$ per octave $(40-80 \mathrm{~Hz})$ with an average slope of $-13 \mathrm{~dB}$ per octave. The slope was $6 \mathrm{~dB}$ per octave between 320 and $500 \mathrm{~Hz}$.

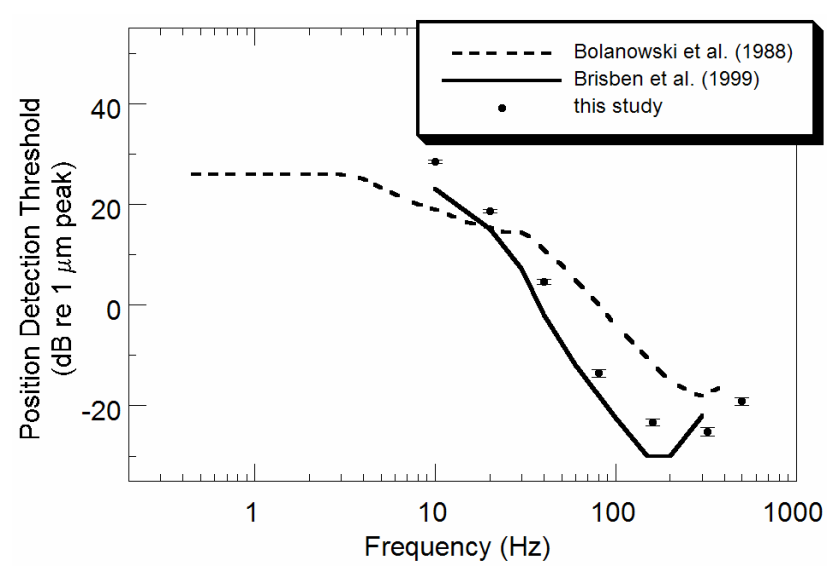

Figure 3. Measured position detection thresholds and comparison to the published detection thresholds.

A two-way ANOVA with frequency and participant as independent variables showed that both the frequency and participant factors as well as their interaction term were significant (for frequency $F(6,350)=17087$, for participants $F(9,350)=287$, interaction term $F(54,350)=57$, all $\mathrm{p}<0.0001)$. In order to examine the possible effect of participants' prior 
experience with using haptic devices, a two factor ANOVA (frequency and experience) was conducted. The results showed no significant difference in threshold between the experienced and inexperienced participants $(F(1,406)=0.49$, $\mathrm{p}=0.48$ ).

The force detection thresholds are shown in Fig. 4 for all participants. The error bars present the standard errors of the means. The shape of the force threshold curve was similar to that of the position threshold curve. The force threshold decreased with the increase of test frequency and exhibited a U-shaped curve at high frequencies after achieving a minimum at around $160 \mathrm{~Hz}$. The slope in the frequency range $10-160 \mathrm{~Hz}$ varied from $-1.6 \mathrm{~dB}$ per octave (between 10 and $20 \mathrm{~Hz}$ ) to $-19.5 \mathrm{~dB}$ per octave (between 40 and $80 \mathrm{~Hz}$ and between 80 and $160 \mathrm{~Hz}$ ) with an average slope of $-12 \mathrm{~dB}$ per octave. The rising slope was $21 \mathrm{~dB}$ between 320 and $500 \mathrm{~Hz}$. Similar to the position threshold data, a two-way ANOVA showed that both the frequency and participant factors as well as the interaction term were significant (for frequency $F(6,350)=3087$, for participants $F(9,350)=251$, interaction term $F(54,350)=57$, all $\mathrm{p}<0.0001)$. The effect of experience was also similar to that exhibited by the position threshold data: not significant $(F(1,406)=0.09, \mathrm{p}=0.76)$.

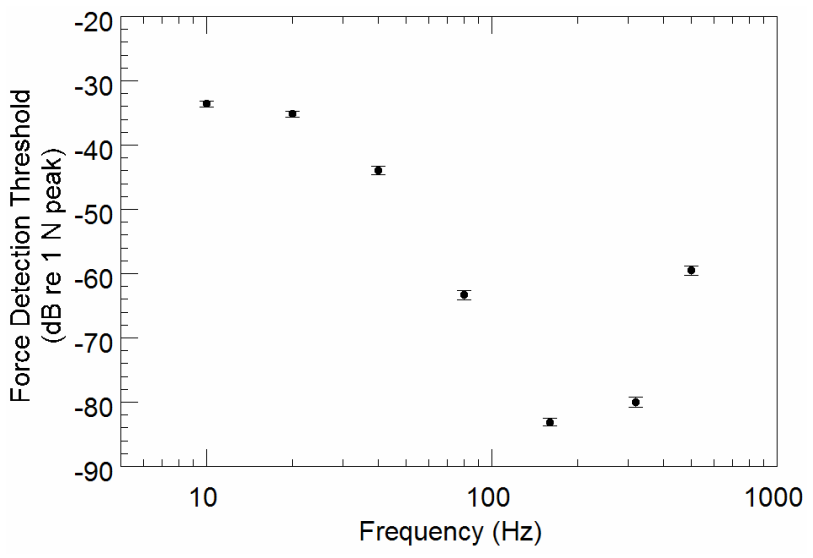

Figure 4. Measured force detection thresholds. Shown are group averages and standard errors.

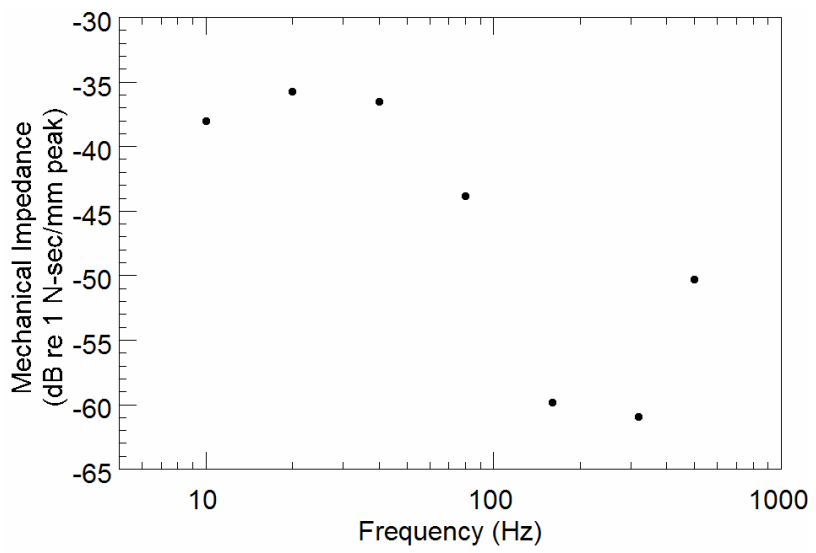

Figure 5. Mechanical impedance of the skin.
The average mechanical impedance of the skin was calculated by the ratio of the average force data (Fig. 4) to the average velocity data at the threshold levels. The impedance of the skin as a function of frequency is presented in Fig. 5. For frequencies below $40 \mathrm{~Hz}$, the mechanical impedance of the skin was roughly constant $(-36$ to $-38 \mathrm{~dB}$ re $1 \mathrm{~N}-\mathrm{sec} / \mathrm{mm}$ peak). It then decreased initially with a slope of $-7 \mathrm{~dB}$ per octave between 40 and $80 \mathrm{~Hz}$ and subsequently with a slope of $-16 \mathrm{~dB}$ per octave between $80-160 \mathrm{~Hz}$. The impedance was almost constant between $160-320 \mathrm{~Hz}$ and then rose with a slope of $16 \mathrm{~dB}$ per octave between $320-500 \mathrm{~Hz}$.

\section{SUMMARY AND DISCUSSION}

The position threshold curve obtained in the present study was similar to those obtained in [6] and [8] (compare curves in Fig. 3). The average slope of the threshold curve in the present study and those of the previous studies are very similar at high frequencies. The lower slope in the midfrequency portion found in Bolanowski et al. [6] was mainly due to the use of a surround around the contact area that restricted the spread of mechanical vibrations across the skin surface $[7,19,20]$. Our threshold data at $10 \mathrm{~Hz}(26 \mu \mathrm{m})$ and $20 \mathrm{~Hz}(8.5 \mu \mathrm{m})$ were above those reported in $[6,8]$. Our lowest threshold level was obtained at $320 \mathrm{~Hz}(0.06 \mu \mathrm{m})$, which was at a higher frequency compared to $150 \mathrm{~Hz}(0.03$ $\mu \mathrm{m})$ obtained in [8] but similar to $300 \mathrm{~Hz}(0.13 \mu \mathrm{m})$ obtained in [6]. The discrepancies in the threshold level are mainly due to the differences in experimental conditions including contact site (multiple sites at the fingerpads and along the sides of digits in the present study, thenar eminence in [6], palm in [8]), contact area (single or multiple contacts), direction of vibration (normal or tangential) and the use of a surround (in [6] but not in [8] or the present study). It has been shown in both [8] and [15] that the fingerpad is more sensitive to tangential vibrations than normal vibrations. In general, the overall shapes of the curves are similar, including a steep slope at around mid/high frequency region and then a Ushaped curve at high frequency region. The most sensitive frequency lies around 200-300 Hz.

The force threshold curve obtained in the present study is perhaps the first of its kind (Fig. 4). The general shape of the force curve was similar to that of the position threshold curve (Fig. 3). The main difference between the force and position threshold curves was that the force curve exhibited a lower slope at low frequencies and a steeper slope at high frequencies. The relationship between the position and force thresholds can be better explained by considering the mechanical impedance derived from them (Fig. 5).

The mechanical impedance of the skin obtained in the present study exhibited all three components of mass, damper and spring as reported in previous studies. The low slope at low frequencies $(<40 \mathrm{~Hz})$ indicated a prominent viscous element. A stiffness component was observed in the midfrequency region ( 40 to $80 \mathrm{~Hz}$ ) by the $-7 \mathrm{~dB}$ per octave slope, and an inertial component was dominant at high frequencies $(>320 \mathrm{~Hz})$ as shown by the rising slope of the curve in Fig. 5 . 
The shape of the mechanical impedance curve (Fig. 5) was very similar to the one reported for the glabrous skin of the hand in [17]. In [17], the low frequency skin impedance ranged from $20-35 \mathrm{~dB}$ re $1 \mathrm{~N}-\mathrm{sec} / \mathrm{m}$ peak and had a resonance frequency (i.e., frequency at the lowest impedance level) between $100-200 \mathrm{~Hz}$ at the glabrous skin of the finger (including distal, middle and proximal phalanges). The amplitude of the impedance at the resonance frequency was about -10 to $+15 \mathrm{~dB}$ re $1 \mathrm{~N}-\mathrm{sec} / \mathrm{m}$ peak. The impedance then increased as the frequency increased. The similarities between the results from the present study and those reported by Lundstrom [17] indicate that the mechanical impedance of the skin is the same for sinusoidal vibrations presented either normal or tangential to the skin.

\section{CONCLUDING REMARKS}

In the present study, haptic force and position detection thresholds for sinusoidal waveforms as well as the impedance of the human hand were evaluated while the participants held a stylus with a pen-hold posture. The low- and high-frequency thresholds differed from previous studies in which vibrations were presented normal to the skin and with an annulus surround around the contact. However, the thresholds were similar to those studies in which vibrations were presented parallel to the skin and in the absence of the surround. The overall shape of the force threshold curve was similar to that of the position threshold curve. The estimated mechanical impedance was dominated by damping effects in the low frequency region and inertial effects in the high frequency region. Overall, the mechanical impedance of the skin associated with tangential vibrations found in the present study was similar to those reported in previous studies using normal vibrations.

The results of the present study have provided muchneeded empirical data on position and force thresholds that have been specifically measured for the use of a stylus attached to the end point of a force-feedback haptic device. They can be used to assess the perceived intensities of proximal stimuli while interacting with a virtual haptic environment through a force-feedback device. We will be using these data in the future for detailed analyses of haptic perception of real and virtual objects. We will also investigate if the mechanical impedance of the skin remains the same with suprathreshold-level vibrations presented in a pen-hold posture. To the extent that the mechanical impedance of the skin depends on stimulation level, we will then investigate trends in variations of the mechanical impedance as a function of stimulus amplitude, in an effort to better understand the interaction between the skin and proximal stimulus. Such modeling efforts will be useful for the design of new haptic interfaces in the future.

\section{ACKNOWLEDGMENT}

The authors wish to thank Roy B. Chung, Chanon M. Jones and Monica Siepmann for their assistance with debugging and data collection and analysis. The second author was a post-doctoral research associate in the Haptic Interface Research Laboratory at Purdue University when this work was initiated. This work was supported in part by research grant No. R01-DC00126 from the National Institute on Deafness and Other Communication Disorders, National Institutes of Health, and in part by a National Science Foundation award under Grant 0098443-IIS.

\section{REFERENCES}

[1] S. Choi and H. Z. Tan, "Toward realistic rendering of haptic textures," IEEE Computer Graphics and Applications, vol. 24, pp. 40-47, 2004.

[2] S. Choi and H. Z. Tan, "Perceived instability of virtual haptic texture. I. Experimental studies," Presence, vol. 13, pp. 395-415, 2004.

[3] S. Choi and H. Z. Tan, "Perceived instability of virtual haptic texture. II. Effect of collision detection algorithm," Presence, vol. 14, pp. 463-481, 2005.

[4] S. Choi and H. Z. Tan, "Perceived instability of virtual haptic texture. III. Effect of update rate," Presence (in press), 2006.

[5] S. Belloni, A. Formaglio, G. Menegaz, H. Z. Tan, D. Prattichizzo, and M. Barni, "Is haptic watermarking worth it?," in Proceedings of the Human Vision and Electronic Imaging XI, pp. 15-19, 2006.

[6] S. J. Bolanowski, G. A. Gescheider, R. T. Verrillo, and C. M. Checkosky, "Four channels mediate the mechanical aspects of touch," The Journal of the Acoustical Society of America, vol. 84, pp. 1680-1694, 1988.

[7] R. T. Verrillo and G. A. Gescheider, "Perception via the sense of touch," in Tactile Aids for the Hearing Impaired, I. R. Summers, Ed. London: Whurr Publishers, 1992, pp. 1-36.

[8] A. J. Brisben, S. S. Hsiao, and K. O. Johnson, "Detection of vibration transmitted through an object grasped in the hand," Journal of Neurophysiology, vol. 81, pp. 1548-1558, 1999.

[9] H. Levitt, J. M. Pickett, and R. A. Houde, "Sensory Aids for the Hearing Impaired." New York: IEEE Press, 1980.

[10] H. Z. Tan and A. Pentland, "Tactual displays for sensory substitution and wearable computers," in Fundamentals of Wearable Computers and Augmented Reality, W. Barfield and T. Caudell, Eds. Mahwah, NJ: Lawrence Erlbaum Associates, 2001, pp. 579-598.

[11] T. H. Massie and J. K. Salisbury, "The PHANTOM haptic interface: A device for probing virtual objects," in Proceedings of the the 3rd Symposium on Haptic Interfaces for Virtual Environment and Teleoperator Systems, pp. 295-301, 1994.

[12] S. Grange, F. Conti, P. Helmer, P. Rouiller, and C. Baur, "Delta Haptic Device as a nanomanipulator," in Proceedings of the SPIE Microrobotics and Microassembly III, pp. 100-111, 2001.

[13] S. Yang, H. Z. Tan, P. Buttolo, and M. Johnston, "Detection of Torque Vibrations Transmitted Through a Passively-Held Rotary Switch," in Proceedings of the Eurohaptics, pp. 217-222, 2004.

[14] A. Z. Hajian and R. D. Howe, "Identification of the Mechanical Impedance at the Human Finger Tip," ASME Journal of Biomechanical Engineering, vol. 119, pp. 109-119, 1997

[15] J. Biggs and M. A. Srinivasan, "Tangential Versus Normal Displacements of Skin: Relative Effectiveness for Producing Tactile Sensations," in Proceedings of the 10th Symposium on Haptic Interfaces for Virtual Environment and Teleoperator Systems, pp. 121-128, 2002.

[16] T. J. Moore and J. R. Mundie, "Measurement of Specific Mechanical Impedance of the Skin: Effects of Static Force, Site of Stimulation, Area of Probe, and Presence of a Surround," The Journal of the Acoustical Society of America, vol. 52, pp. 577$584,1972$.

[17] R. Lundstrom, "Local Vibrations - Mechanical Impedance of the Human Hand's Glabrous Skin," Journal of Biomechanics, vol. 17, pp. 137-144, 1984.

[18] R. G. Dong, D. E. Welcome, T. W. McDowell, and J. Z. Wu, "Biodynamic Response of the Human Fingers in a Power Grip Subjected to a Random Vibration," ASME Journal of Biomechanical Engineering, vol. 126, pp. 447-457, 2004.

[19] A. Israr, P. H. Meckl, and H. Z. Tan, "A two DOF controller for a multi-finger tactual display using a loop-shaping technique," in Proceedings of the ASME International Mechanical Engineering Congress and Exposition (IMECE04), pp. 1083-1089, 2004

[20] C. L. Van Doren, "The effects of a surround on vibrotactile thresholds: evidence for spatial and temporal independence in the non-Pacinian I (NP I) channel," The Journal of the Acoustical Society of America, vol. 87, pp. 2655-2661, 1990.

[21] H. Levitt, "Transformed up-down methods in psychoacoustics," The Journal of the Acoustical Society of America, vol. 49, pp. 467-477, 1971.

[22] M. R. Leek, "Adaptive procedures in psychophysical research," Perception and Psychophysics, vol. 63, pp. 1279-1292, 2001. 